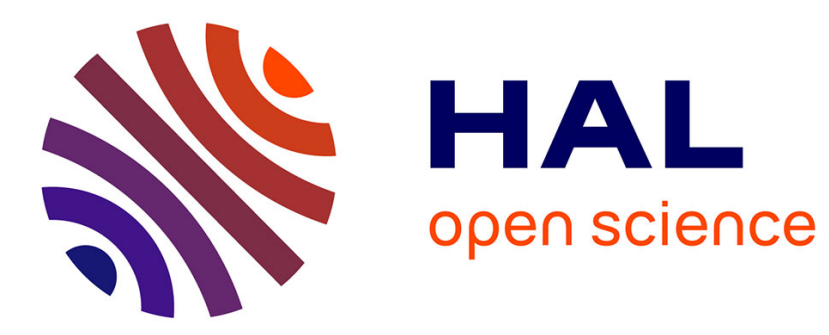

\title{
Discovering Emergent Behaviors from Tracks Using Hierarchical Non-parametric Bayesian Methods
}

\author{
Guillaume Chiron, Petra Gomez-Krämer, Michel Ménard
}

\section{To cite this version:}

Guillaume Chiron, Petra Gomez-Krämer, Michel Ménard. Discovering Emergent Behaviors from Tracks Using Hierarchical Non-parametric Bayesian Methods. ICPR - 22nd International Conference on Pattern Recognition, Aug 2014, Sweden. pp.2185, 10.1109/ICPR.2014.380 . hal-01062000

\section{HAL Id: hal-01062000 https://hal.science/hal-01062000}

Submitted on 9 Sep 2014

HAL is a multi-disciplinary open access archive for the deposit and dissemination of scientific research documents, whether they are published or not. The documents may come from teaching and research institutions in France or abroad, or from public or private research centers.
L'archive ouverte pluridisciplinaire HAL, est destinée au dépôt et à la diffusion de documents scientifiques de niveau recherche, publiés ou non, émanant des établissements d'enseignement et de recherche français ou étrangers, des laboratoires publics ou privés. 


\section{Discovering Emergent Behaviors from Tracks Using Hierarchical Non-parametric Bayesian Methods}

\author{
Guillaume Chiron \\ L3i, University of La Rochelle, \\ La Rochelle 17071 Cedex 9, France \\ Email: guillaume.chiron@univ-lr.fr
}

\author{
Petra Gomez-Krämer \\ L3i, University of La Rochelle, \\ La Rochelle 17071 Cedex 9, France \\ Email: petra.gomez@univ-lr.fr
}

\author{
Michel Ménard \\ L3i, University of La Rochelle, \\ La Rochelle 17071 Cedex 9, France \\ Email: michel.menard@univ-lr.fr
}

\begin{abstract}
In video-surveillance, non-parametric Bayesian approaches based on a Hierarchical Dirichlet Process (HDP) have recently shown their efficiency for modeling crowed scene activities. This paper follows this track by proposing a method for detecting and clustering emergent behaviors across different captures made of numerous unconstrained trajectories. Most HDP applications for crowed scenes (e.g. traffic, pedestrians) are based on flow motion features. In contrast, we propose to tackle the problem by using full individual trajectories. Furthermore, our proposed approach relies on a three-level clustering hierarchical Dirichlet process able with a minimum a priori to hierarchically retrieve behaviors at increasing semantical levels: activity atoms, activities and behaviors. We chose to validate our approach on ant trajectories simulated by a Multi-Agent System (MAS) using an ant colony foraging model. The experimentation results have shown the ability of our approach to discover different emergent behaviors at different scales, which could be associated to observable events such as "forging" or "deploying" for instance.
\end{abstract}

\section{INTRODUCTION}

There is an increasing demand in automated visual surveillance systems. In recent years, mining activities from crowded scenes (e.g. traffic, pedestrians) have become an active research topic. The insect and animal behavior community is also following the track with closely related issues. Recovering and modeling the evolution of an insect colony activity opens interesting perspectives for biologists. For instance, current studies focus on environmental monitoring applications based on behavioral information of bio-indicator species (e.g. honeybees [1]). Studying the activity of an insect colony is a different problem compared to studying human activity, in terms of speed, number and the chaotic motion of the targets. Contrary to crowded scenes frequently used in video-surveillance such as traffic or pedestrian scenes, trajectories generated by an insect colony are naturally less limited by environmental constraints (e.g. walls, corridors, roads, circulation rules), and sometimes even do not follow any perceptible trends.

The recent growth of RGB-D cameras offers new possibilities for recovering individual 3D tracks from insect colonies or swarms ([2], [3], [4]). At a global level, we consider the complete chain made of the following steps: acquisition, segmentation, tracking, and behavior analysis. This paper essentially focuses on the last step by proposing an approach for discovering emergent behaviors from a massive number of unconstrained trajectories. The purpose of our work is to model behaviors with a minimum a priori on the model. Non-parametric Bayesian approaches based on a Hierarchical
Dirichlet Process (HDP) have recently shown the efficiency of modeling crowed scene activities. Most HDP applications for crowed scenes are based on flow motion features (see Section II). In contrast, we propose to tackle the problem by working on individual full trajectories (sequence of points).

Our proposed approach works from trajectory datasets computed from videos. A dataset is structured into captures and subclips. Using a multi-layer clustering HDP, notable events are detected at different semantic levels. Then, captures and subclips are labeled with the most representative events relatively to its level. Our model is made of the three following semantic levels (detailed further): behavior, activity, and activity atoms. Similar concepts have been presented in [5] with the Dual-HDP, and in [6] with the MLC-HDP but for a medical application.

Despite the existing approaches for recovering insect tracks, to our knowledge no ground truth is available at the scale of behaviors. Therefore, we chose to validate our approach with ant trajectories simulated by a Multi-Agent System (MAS) using an ant colony foraging model, which has the advantage compared to other simulation methods, to provide trajectories which does not follow any direct parametric model. The modeling approach based on the MLC-HDP and the validation methods are illustrated by Figure 1. The code that associates colored circles and squares to behaviors and activities respectively will be kept for the rest of the paper.

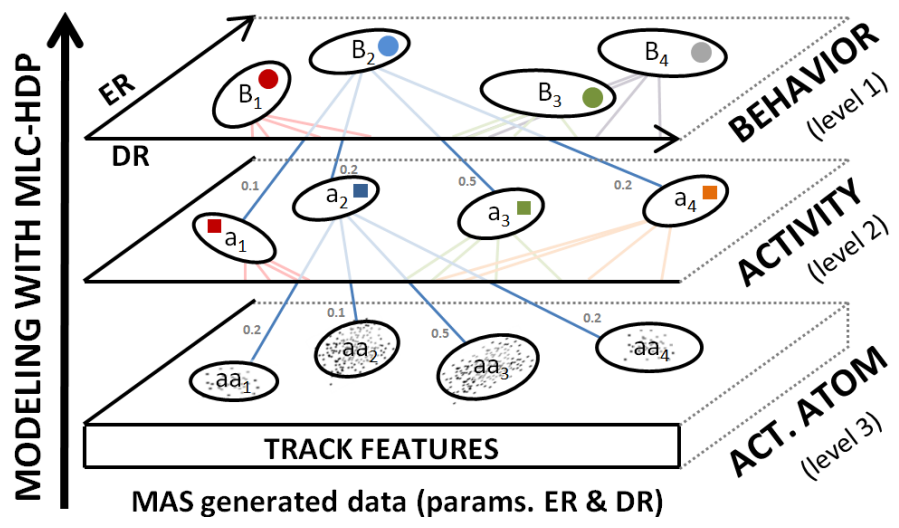

Fig. 1. Behavioral modeling approach validated by a MAS simulated dataset. The MLC-HDP clusterises trajectories at increasing semantic levels from the "Activity atom" to the "Behavior" level. This allows to visualize the effect of the MAS parameters on the recovered behaviors. The parameters ER \& DR are defined in Section IV. 
In summary, this paper presents an original approach using a minimum a priori for discovering emergent behaviors from a structured trajectory dataset, leading to the three following contributions:

- The use of a three-layered HDP to discover emergent behaviors. As far as we know, similar approaches related to activity modeling are using a maximum of two 2 layers.

- The use of features extracted from individual trajectories, rather than flow motion based features commonly used for crowded scenes.

- The original validation by a MAS simulation, indirectly providing the semantic ground truth associated to the dataset.

This paper is organized as follows. First, Section II details the background and related work in the domain of modeling activity and behavior. Section III introduces the method based on MLC-HDP, and details the features extracted from trajectories. Then, Section IV validates our method using simulated ant trajectories. Finally, Section V concludes our work and opens promising perspectives for modeling insect activity and behavior.

\section{BACKGROUND AND RELATED WORK}

According to the nature of the dataset, and the level of observation needed, different approaches have been proposed in the literature for modeling activities and behaviors.

A first approach, largely studied in the insect and animal behavior community, focuses on individual tracks. It consists in modeling the behaviors of each individual target based on local trajectory features. Then, more or less advanced clustering or classification methods are used in order to recognize behaviors. For example, in [7], 2D honeybee trajectories were classified using a Hidden Markov Model (HMM). In [8] a clustering based hierarchical classifier is used to separate fish trajectories in a normal and abnormal class. Some methods ([9] and [10]) even join behavior analysis to the tracking in order to improve its efficiency.

A second approach is oriented on mass (or swarm) activity modeling. In general, due to the nature of crowded scenes, the analysis of the activity relies on optical flow motion features. For example, in [11] a bag of word classifier is used to detect and localize abnormal crowd activities relying on sparse optical flow features. In [12] a combined HMM and spectral clustering are used for detecting abnormal crowd scenarios. In [13] the overall behavior of the scene is characterized by modeling the motion variation of local space-time volumes and their spatialtemporal statistical behaviors.

However, the domain of colony (or swarm) activity modeling of has not been much less explored in the insect and animal behavior community. Indeed, due to the small, fast and chaotic nature of the targets, classical approaches based on optical flow motion features have been discarded. But recently, papers such as [4], [14], and [2] propose massive multiple target tracking methods for insects, and open interesting perspectives in term of activity modeling.

Hierarchical Bayesian models provide an attractive solution for modeling chaotic activity. The Hierachical Dirichlet Process (HDP) originally introduced in [15] is a non-parametric Bayesian approach for clustering a dataset on multiple levels and sharing information across a hierarchy. It has been widely used in the document community (documents/topics analysis). Recently HDP based approaches have shown their efficiency in clustering structured data in other domains. Briefly, the Dual-HDP presented in [5] consists in cross-clustering atomic activities of sub-video clips extracted from a video in order to find co-occurring activities. Then, in the medical domain, in [16] tractographies (data closely similar to trajectories) are segmented using a HDP mixture model. Also, in [6], the multi-level clustering HDP method (another extension of HDP) is proposed for clustering for human electroencephalography seizure modeling at different levels.

\section{PROPOSED APPROACH}

This section details the proposed approach for discovering emergent behaviors from trajectories using a hierarchical nonparametric Bayesian method: the Hierarchical Multi-Layer Clustering Dirichlet Process (MLC-HDP). This section is organized in four parts. First, the structure of the dataset and the feature extraction are presented. Then, a reminder of the classic Dirichlet Process (DP) is made. And, finally the use of the MLC-HDP adapted to our problem is detailed.

\section{A. Dataset Structure}

The structure of the dataset used in our approach is as follows. We consider several videos capturing the same environment at different times using the same point of view. On those videos, a tracking method could be applied (e.g. [2]) to recover the target trajectories (sequence of points). The datasets used in Section IV are generated by a MAS, which bypass the tracking step. In the following we consider a capture as a temporal ordered set of trajectories. Every capture is segmented into subclips using a non-overlapping sliding window (e.g. 5 seconds). The size of the window should be big enough to ensure a sufficient number of trajectories in each subclip, but should also be small enough to avoid multiple distinct activities occurring during a unique subclip. Examples of activities are given later. Therefore the dataset is constituted of numerous trajectories structured into captures and subclips configured with a given granularity. Figure 2 illustrates an example of the structure of the dataset.

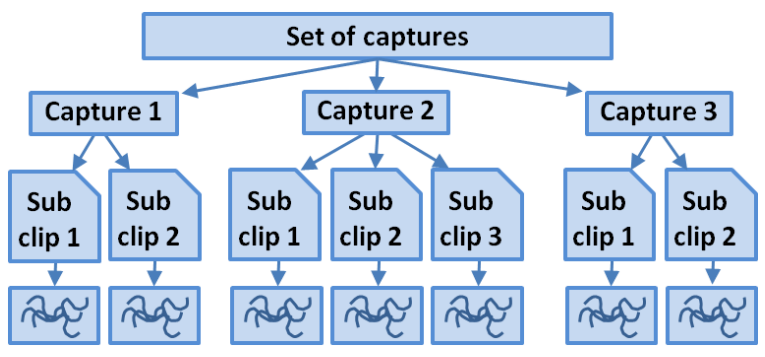

Fig. 2. Example of structured dataset.

\section{B. Feature Extraction}

The features extracted from trajectories constitute the base of the clustering mechanism. As an example, the authors of [8] represent fish trajectories by 179 features (originally 776 features extracted, then reduced by Principal Component Analysis (PCA)). Our approach is inspired from that work, 
namely by considering a mix of local and environmental related characteristics. In practice, living entity trajectories are chaotic and hardly follow distinguishable patterns. That explains the need of many features for representing a track, especially for long tracks. However, as we focus on detecting notable events rather than detecting abnormal trajectories among normal trajectories, a limited number of features is sufficient. For each trajectory, a vector made of the following features is extracted. It represents the trajectory by a point defined in $\mathbb{R}^{d}$. Finally, all vectors are normalized over each dimension individually.

Trajectory relative invariant features: The Centered Distance Function (CDF) mentioned in [17] consists in computing the relative distance between all points of the track and the centroid, given for 2D trajectories by:

$$
c(i)=\sqrt{(x(i)-\bar{x})^{2}+(y(i)-\bar{y})^{2}}
$$

with $i$ the trajectory point index. The 7 following features are extracted from the vector $c$ : mean, standard deviation, min, max, skewness, kurtosis and number of mean crossings.

Environmental absolute features: Considering our 2D case, the scene space is split into a grid of $n$ rows and $m$ columns. A feature set is made of $n \times m$ values (e.g. $6 \times 6=36$ ), corresponding to the time spent by the each target in the corresponding cell. A PCA is applied in order to reduce the number of dimensions while keeping at least $90 \%$ of the information.

\section{Dirichlet Process}

As defined in [16], the DP is used as a prior to sample probability measures. It is defined by a concentration parameter $\alpha$, which is a positive scalar, and a base probability measure $H$. A probability measure $G$ randomly drawn from Dirichlet process $D P(\alpha, H)$ is always a discrete distribution even if $H$ is continuous. $G=\sum_{k=1}^{\infty} \pi_{k} \delta_{\phi_{k}}$ which can be obtained from a stick-breaking construction. In the context of mixture models, a DP-distributed discrete random measure is used as a prior over the parameters of mixture components:

$$
\begin{aligned}
G & \sim D P(\alpha, H) \\
\theta_{i} \mid G & \sim G \\
x_{i} \mid \theta_{i} & \sim F\left(\theta_{i}\right)
\end{aligned}
$$

where $\left\{x_{i}\right\}$ are the observable variables to be modeled, $\theta_{i}$ are the parameters of the mixture component that $x_{i}$ belong to (e.g. mean and unit variance of a Gaussian component), $F$ represents the distribution of mixture components (e.g. Gaussian in a mixture of Gaussian).

\section{Multi-Layer Clustering HDP}

The MLC-HDP has originally been introduced in [6] for a medical application. We adapted this method to our trajectories/behavior modeling problem. Figure 3 illustrates the two incremental extensions (HDP [15] and MLC-HDP) in addition to the classical DP. The classical DP is not able to share clusters across groups of data. The HDP provides clusters that are shared across the different groups of data. The MLCHDP is additionally able to cluster data over multiple layers. Keeping only its bottom layer, the MLC-HDP is equivalent to the HDP.
As illustrated in Figure 1, our approach is constructed over the three following semantic levels, which are associated to different elements of the dataset structure. Examples of events are given relatively to our ant foraging model described in Section IV.

Level 1 - Behavior: A behavior is represented by a set of weights over all the activities. A behavior is associated to each capture. Example: One by one food pile foraging, No foraging.

Level 2 - Activity: An activity is represented by a set of weights over all the activity atoms. An activity is associated to each subclip. Example: Foraging pile $p$, Deploy after foraging pile $p$.

Level 3 - Activity atom: An activity atom is a cluster of similar trajectories regarding local features extracted from individual trajectories. Example: Group of ants walking in the same direction.

As the dataset is ordered in time, it is possible to discern the semantic evolution at different time scales (between captures or subclips).
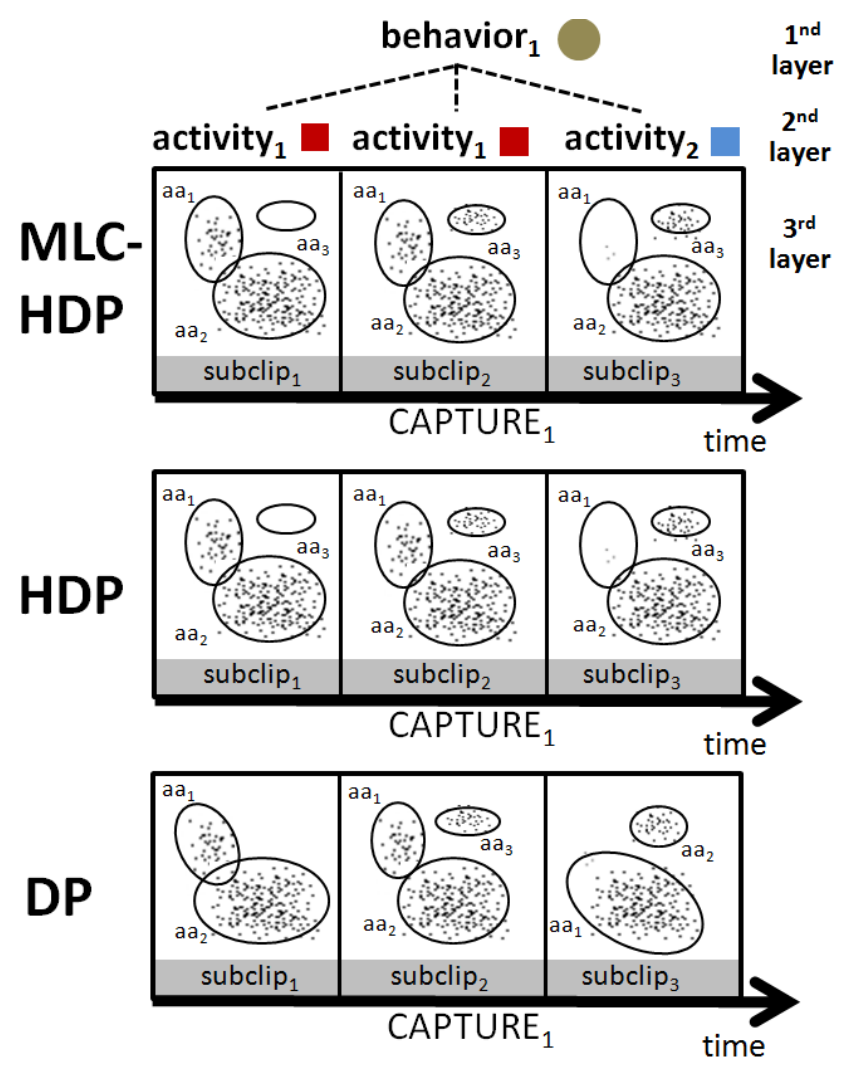

Fig. 3. Examples of clustering with DP, HDP and MLC-HDP over a simple structured dataset.

Formally, we consider a dataset of $T$ videos captured, with each capture $t$ composed of $J_{t}$ subclips, with each subclip $j$ composed of $N_{j i}$ trajectories signatures, which we call $x_{t j i} \in R^{d}$, with $d$ the feature space dimension. The observation $\left\{\left\{\left\{x_{t j i}\right\}_{i=1}^{N_{t j}}\right\}_{j=1}^{J_{t}}\right\}_{t=1}^{T}$ of every trajectory is modeled by a set of unique base-distribution atoms with prior measure $H$ and parameter $\lambda$ for an arbitrary distribution $x_{t i j} \sim F\left(\theta_{t j i}\right)$ where $\theta_{t j i}$ are the parameters of the model, which are equal to those of a unique base-level atom $\phi_{k}$. We consider $F$ to be 
a multivariate Normal distribution with diagonal covariance. The three level MLC-HDP is defined as follows:

$$
\begin{aligned}
& \text { Level 1: Behavior } \\
& \beta^{(1)} \sim \operatorname{GEM}\left(\gamma^{(1)}\right) \\
& \pi^{(1)} \sim D P\left(\alpha^{(1)}, \beta^{(1)}\right) \\
& z_{t}^{(1)} \sim \pi^{(1)}
\end{aligned}
$$

Level 2: Activity

$$
\beta^{(2)} \sim G E M\left(\gamma^{(2)}\right)
$$$$
\pi_{l}^{(2)} \sim D P\left(\alpha^{(2)}, \beta^{(2)}\right)
$$$$
z_{t j}^{(2)} \sim \pi_{l=z_{t}^{(1)}}^{(2)}
$$

\section{Level 3: Activity atom}

$$
\beta^{(3)} \sim \operatorname{GEM}\left(\gamma^{(3)}\right)
$$

$\pi_{\ell}^{(3)} \sim D P\left(\alpha^{(3)}, \beta^{(3)}\right)$

$z_{t j i}^{(3)} \sim \pi_{\ell=z_{t j}^{(2)}}^{(3)}$
Trajectory feature

$\phi_{k} \sim H(\lambda)$

$x_{t i j} \sim F\left(\phi_{k=z_{t j i}^{(3)}}\right)$ with $G E M$ as the Griffiths-Engen-McCloskey distribution. The MLC-HDP hyper-parameters a priori $\gamma$ and $\alpha$ are defined manually (or can be sampled from a Gamma law). The intensity parameter $\alpha$ regulates the number of spikes. More details on the MLC-HDP model can be found in [6].

In addition, the Figure 4 illustrates the inference mechanisms, which makes the link between the model and the dataset. Namely, the parameters of the model at different levels (set of weights $\pi$ and $\beta$, and cluster membership indexes $z$ ) are refined by performing iterative sampling until the model is considered to fit enough the data. The parameters $\phi_{k}=\left(\mu_{k}, \sigma_{k}\right)$ for each of the $K$ activity atoms (or base atoms) are sampled according to the trajectory features data, with the particularity to always add an empty cluster (sampled from the prior $H(\lambda)$ ) when the last empty one has been associated to some data.

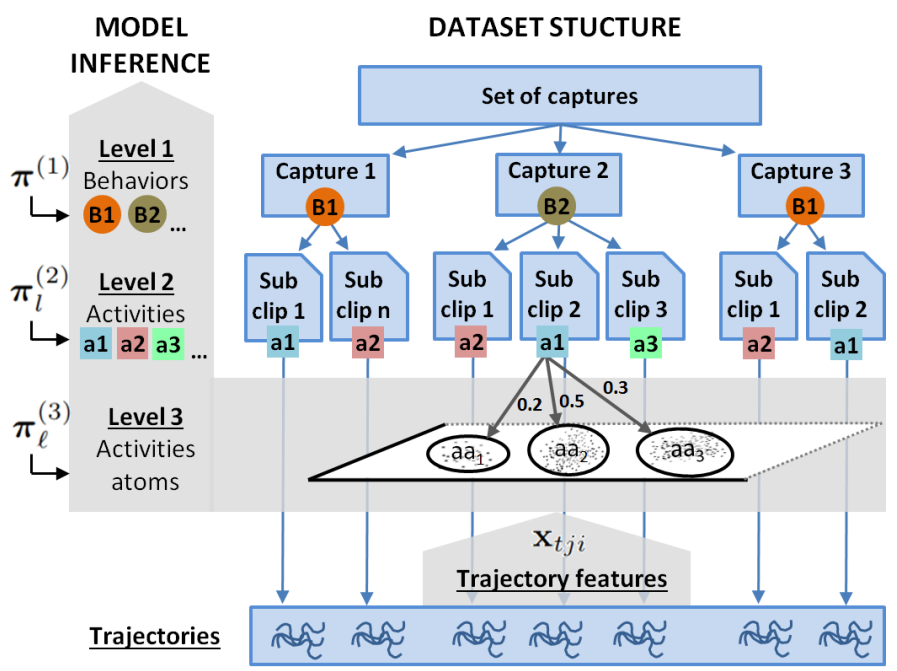

Fig. 4. Inference mechanism of the model parameters from the dataset. In descending order, the blue boxes show the structure of the trajectory dataset. In ascending order, the grey arrows represent the step by step modeling of the data until the behavior level.

\section{VALIDATION}

In this section, we validate our approach by running the MLC-HDP on ant trajectories generated by a MAS. The advantage of validating our behavior modeling with a MAS is twofold. First, MAS models can generate emergent behaviors with a relatively low number of parameters. Different sets of these parameters lead to different behaviors. Second, simulated trajectories are not directly constraint by any trajectory model. The interesting point is that both sides (simulation and modeling) are used with a minimum a priori. The effect of the MAS parameters can be observed on the top behavior layer.

We chose a model that simulates the foraging of an ant colony (implementation given in [18]). This model is driven by the following parameters: the environment (location of the nest and different food sources), the population, the Diffusion Rate (DR) and the Evaporation Rate (ER) of the pheromones secreted by the ants. Figure 5 illustrates the MAS simulation under four different configurations. The effect of the DR and EP parameters are clearly observable on the food pile graphs. The configuration A (low DR, average ER) and B (high DR, average ER) leads the ants to forage the food sources one by one. In opposite, in the configurations C (high DR, low ER) and D (high DR, high ER), food piles are foraged simultaneously, but at different efficiencies. Furthermore, we recognize on the Netlogo view that according to the configuration, more or less ants wander around without any purpose.
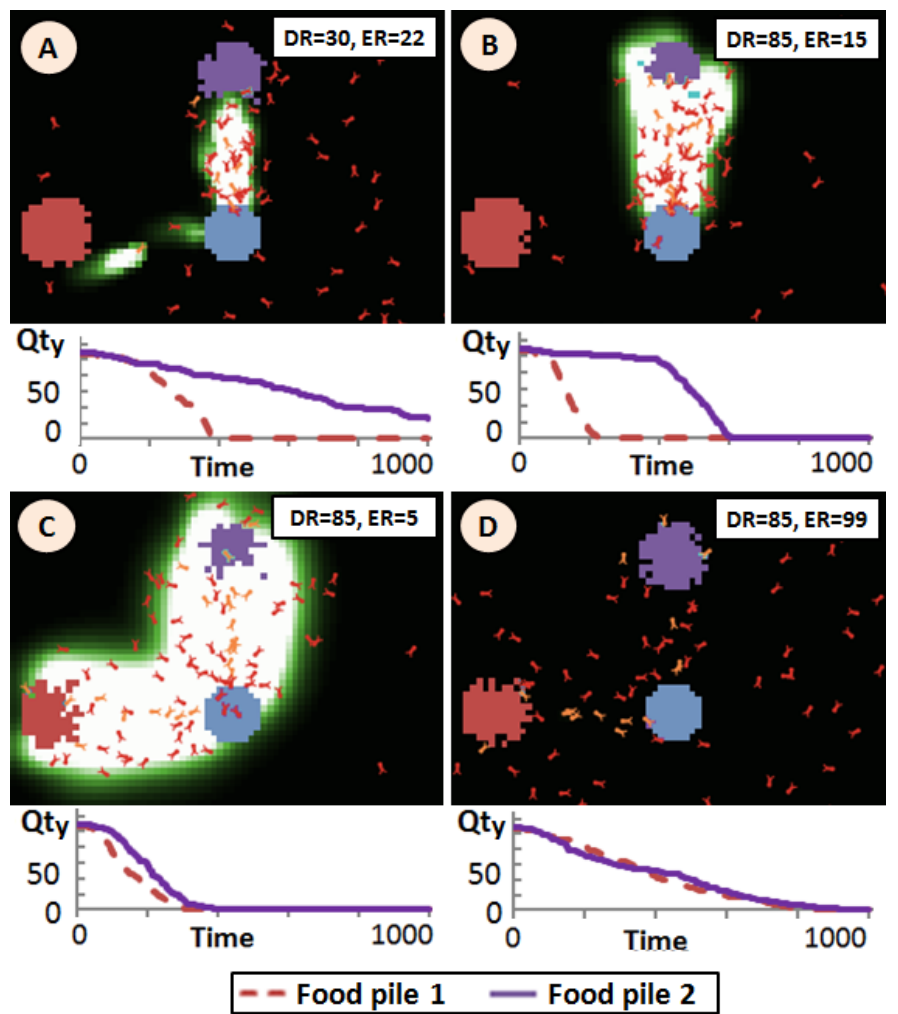

Fig. 5. MAS ant foraging model simulated under four configurations (A,B,C and D), each having a different set of parameters (DR and ER). Each configuration is illustrated by the Netlogo view at the 150th iteration (at the top), and a graphic of the food piles evolution (at the bottom). For each view, the center circle represents the nest, the left and top circles represent the food sources, the red spots represent the ants, and the green/white rainbow represents the intensity of the pheromones secreted by the ants.

The MAS model animates a fixed number of agents into a closed world. From those agents, trajectories are collected to build the dataset that validates our MLC-HDP based approach. During the simulation, each agent generates a varying number of trajectories. For each agent, a trajectory is considered 
between every couple of the following elements: the nest, any food sources, any border of the world. Whatever their situation (wandering, following pheromones), every agents finally always hit one of these elements, which leads to an average trajectories length of 77 points (1 point per time unit).

The couple "Netlogo view" and "food piles evolution graphs" constitutes our ground truth corresponding to the activity layer of MLC-HDP. Additionally, the MAS model parameters (ER and DR) give more information on the "Behavior" layer.

\section{A. MLC-HDP on a Single Capture}

This section illustrates the use of the MLC-HDP on a single capture. As this experiment concerns only one capture, the presented results correspond to the activity layer of the MLCHDP (level 2). Results are shown in Figure 6. In this example, the following parameters have been used for the simulation: $\mathrm{DD}=75, \mathrm{ER}=10$, three food sources disposed at a slightly increasing distance around the nest. The food piles evolution graphic shows that the food sources have been foraged one by one. The simulation lasts 1500 iterations (or time units), and have been discretized into 31 subclips. Each subclip has been associated to one of the 10 distinct activities that have been found by the MLC-HDP. Those 10 activities are models according to 12 activity atoms given different weights. The less and the most represented activity atoms are associated to 49 and 253 trajectories respectively, among a total number of 1550 trajectories. As shown in Figure 6, most of activity changes can be associated to a real event that occurred in the simulation. We associated 7 interpretations to the activities found by the MLC-HDP (e.g. Foraging a food pile, Deploy after foraging). Some activities are uniquely represented (e.g. the 1st, 2nd and 16th from the left). According to the granularity of the dataset, notable events can be mixed and then give birth to a new activity.

\section{B. MLC-HDP on Multiple Captures}

This section illustrates the use of the MLC-HDP on multiple captures. In contrast to the single capture dataset used previously, the following results are focused essentially on the behavior layer of the MLC-HDP (level 1). Nevertheless the success of clustering at the top level of the HDP witnesses the good job of the lower levels. In this experiment, the four different behaviors previously illustrated in Figure 5 are tested. Each of the 4 behaviors are simulated 5 times, which corresponds to 20 captures. The environment ( 2 food sources) and the population (100 ants) parameters are kept stable for all the captures. So the dataset used is composed of 49882 trajectories split in 20 captures, with each capture segmented into 16 subclips. Figure 7 shows the clustering results (at the behavior and activity levels) for the simulated dataset. The twofold strength of the MLC-HDP is clear. First, it has found 4 main behavior categories. Second, it has correctly categorized 18 over 20 captures in adequacy with the model parameters. The wrong categorized captures could be explained by the randomness of the MAS simulation, which could sometimes lead to results at the edge of the category models found by the MLC-HDP. The activity level shows a sequence of activities, which represent the temporal evolution of the colony. It is interesting to notice that this temporal aspect is not taken in

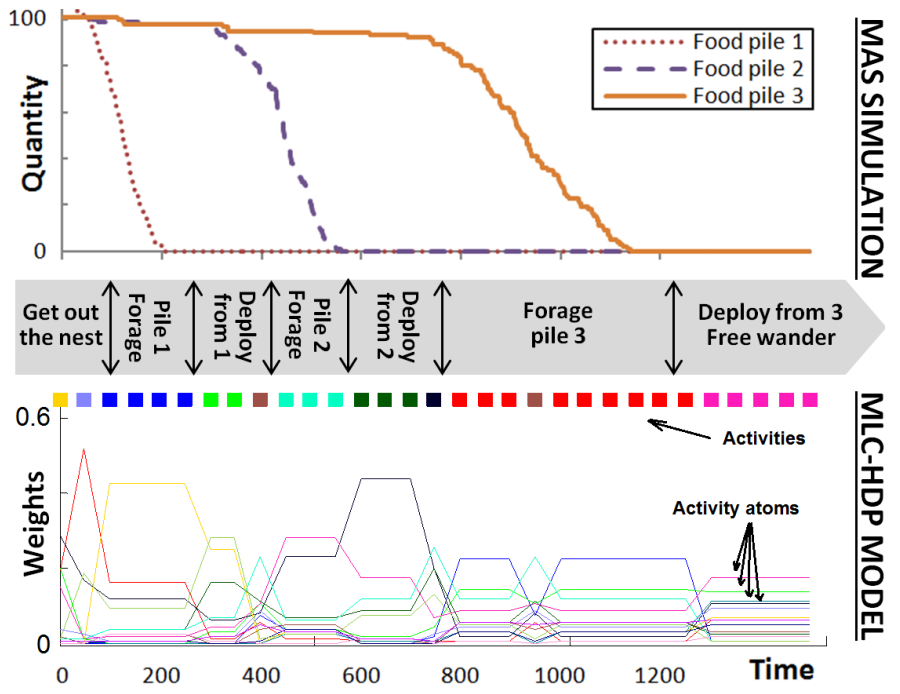

Fig. 6. Activities (clusters of activity atoms) found by the MLC-HDP for a single capture (at the bottom). Each square shows by its color the activity corresponding to the subclip associated. The dataset used in this experiment has been generated by the ant foraging MAS (with parameters $\mathrm{DD}=75, \mathrm{ER}=10$, 3 food sources), and is represented by the food piles evolution graph (at the top). This graph shows notable events (e.g. foraging begin/end). A matching between the notable events (in the food piles evolution graph) and the activity returned by the MLC-HDP is made on a time arrow (in the middle).

account in our approach. For example the "Foraging piles one by one" behavior is unique regardless which pile is foraged first.

\section{CONClusion}

First, this paper has presented an original approach for discovering emergent behaviors from a structured trajectory dataset. To our knowledge, state-of-the-art HDP based methods related to activity modeling used at most two layers. We successfully adapted the MLC-HDP using three layers which was originally designed for clustering medical data (EEG signals) to our problem. Second, most of those existing approaches dealing with crowded scenes use motion flow features. We proposed an alternative based on full trajectories. Finally, we validated our approach using a ant foraging MAS simulation. The MAS and the MLC-HDP both answered our initial requirement of building an approach with models following a minimum a priori.

The results presented in Section IV have shown the ability of our approach to discover different emergent behaviors at different scales. The first experiment performed at the activity scale provided results in coherence with the food pile evolution graph, notable events were clearly explainable from a human point of view (e.g. foraging a pile). The second experimentation has demonstrated the ability of the MLCHDP to approximate the number of behaviors and to classify satisfyingly the captures among these behaviors (e.g. foraging piles one by one) with a minimum a priori.

In terms of perspectives concerning the application, as mentioned in the introduction, the honeybee is a bio-indicator species and catches the interest of biologists. Our team is currently building up a honeybee trajectory dataset following the method detailed in [2]. It would be interesting to extract behavioral information using the proposed method. Concerning 


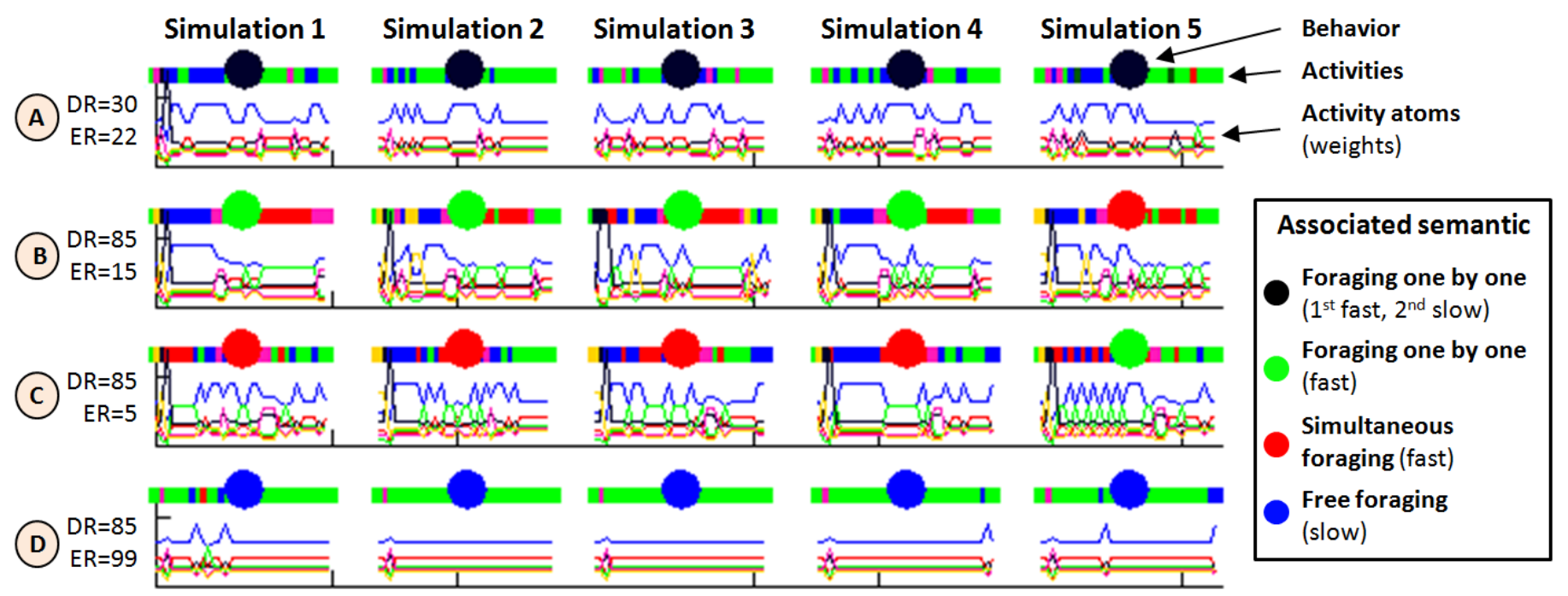

Fig. 7. Results of the MLC-HDP on 20 captures, each composed of up to 2000 ant tracks. Four sets of parameters are experienced, with each set of parameters (ER \& DR) tested on 5 simulations to ensure the reliability. The color of the big circles on top of each capture represents the behavior corresponding to the capture (MLC-HDP lvl1). The smaller squares on top of each capture represent the activities corresponding to the subclips (MLC-HDP lvl2). The colored curves below represent for each subclip, the weights associated to the all the exiting activity atoms (MLC-HDP lvl3).

the methods, in the future we could consider to add a Hidden Markov Model at the top level to model the temporal transition between activities and behaviors. Similar approaches have proposed in [19]. Moreover, as we reach an high level of semantic, we could also consider to label the behavior (as they are limited in number), and used them to link the data to some ontological models.

\section{ACKNOWLEDGMENT}

The authors would like to thank Dr. Drausin Wulsin for making available an implementation of its MLC-HDP (introduced in [6]) and for sharing his expertise regarding our application. This work was supported by FranceAgrimer (contract: RISQAPI, 14-03R) and the Poitou-Charente region (EPERAS project).

\section{REFERENCES}

[1] O. Lambert, M. Piroux, S. Puyo, C. Thorin, M. Larhantec, F. Delbac, and H. Pouliquen, "Bees, honey and pollen as sentinels for lead environmental contamination," Environmental Pollution, vol. 170, pp. 254-259, 2012.

[2] G. Chiron, P. Gomez-Krämer, and M. Ménard, "Detecting and tracking honeybees in 3D at the beehive entrance using stereo vision," EURASIP Journal on Image and Video Processing, vol. 2013, no. 1, p. 59, 2013.

[3] A. D. Straw, K. Branson, T. R. Neumann, and M. H. Dickinson, "Multicamera real-time three-dimensional tracking of multiple flying animals," Journal of The Royal Society Interface, vol. 8, no. 56, pp. 395-409, 2011.

[4] D. Theriault, Z. Wu, N. Hristov, S. Swartz, K. Breuer, T. Kunz, and M. Betke, "Reconstruction and analysis of 3D trajectories of Brazilian free-tailed bats in flight," CS Department, Boston University, Tech. Rep., 2010.

[5] X. Wang, X. Ma, and W. E. L. Grimson, "Unsupervised activity perception in crowded and complicated scenes using hierarchical bayesian models," Pattern Analysis and Machine Intelligence, IEEE Transactions on, vol. 31, no. 3, pp. 539-555, 2009.

[6] D. Wulsin, S. Jensen, and B. Litt, "A hierarchical Dirichlet process model with multiple levels of clustering for human EEG seizure modeling," in 29th International Conference on Machine Learning (ICML), 2012.
[7] A. Feldman and T. Balch, "Representing honey bee behavior for recognition using human trainable models," Adaptive Behavior - Animals, Animats, Software Agents, Robots, Adaptive Systems, vol. 12, no. 3-4, pp. 241-250, 2004.

[8] C. Beyan and R. B. Fisher, "Detection of abnormal fish trajectories using a clustering based hierarchical classifier," in British Machine Vision Conference (BMVC), Bristol, UK, 2013.

[9] A. Veeraraghavan, R. Chellappa, and M. Srinivasan, "Shape-andbehavior encoded tracking of bee dances," IEEE Transactions on Pattern Analysis and Machine Intelligence, vol. 30, no. 3, pp. 463-476, 2008.

[10] J. Campbell, L. Mummert, and R. Sukthankar, "Video monitoring of honey bee colonies at the hive entrance," Visual observation \& analysis of animal \& insect behavior (ICPR), 2008.

[11] R. Mehran, A. Oyama, and M. Shah, "Abnormal crowd behavior detection using social force model," in Computer Vision and Pattern Recognition, 2009. CVPR 2009. IEEE Conference on. IEEE, 2009, pp. 935-942.

[12] E. L. Andrade, S. Blunsden, and R. B. Fisher, "Modelling crowd scenes for event detection," in Pattern Recognition, 2006. ICPR 2006. 18th International Conference on, vol. 1. IEEE, 2006, pp. 175-178.

[13] L. Kratz and K. Nishino, "Anomaly detection in extremely crowded scenes using spatio-temporal motion pattern models," in Computer Vision and Pattern Recognition, 2009. CVPR 2009. IEEE Conference on. IEEE, 2009, pp. 1446-1453.

[14] T. Kimura, M. Ohashi, R. Okada, and H. Ikeno, "A new approach for the simultaneous tracking of multiple honeybees for analysis of hive behavior," Apidologie, vol. 42, no. 5, pp. 607-617, 2011.

[15] Y. W. Teh, M. I. Jordan, M. J. Beal, and D. M. Blei, "Hierarchical Dirichlet processes," Journal of the american statistical association, vol. 101, no. 476, 2006.

[16] X. Wang, W. E. L. Grimson, and C.-F. Westin, "Tractography segmentation using a hierarchical Dirichlet processes mixture model," NeuroImage, vol. 54, no. 1, pp. 290-302, 2011

[17] F. I. Bashir, A. A. Khokhar, and D. Schonfeld, "View-invariant motion trajectory-based activity classification and recognition," Multimedia Systems, vol. 12, no. 1, pp. 45-54, 2006.

[18] U. Wilensky. (1997) Netlogo ants model. Center for connected learning and computer-based modeling, Northwestern University, Evanston, IL. [Online]. Available: http://ccl.northwestern.edu/netlogo/models/Ants/

[19] Q. Gao and S. Sun, "Trajectory-based human activity recognition with hierarchical Dirichlet process hidden Markov models," in Proceedings of the 1st IEEE China Summit and International Conference on Signal and Information Processing, 2013, pp. 1-5. 